\title{
Comparison of rapid and conventional methods for investigating of $m e c A$ presence in Staphylococcus Species
}

\author{
Selim Gorgun ${ }^{1}$, Hacer Isler ${ }^{2}$, Mehmet Cenk Turgut ${ }^{3}$
}

\begin{abstract}
Objectives: Taking the determination of mecA gene by polymerized chain reaction (PCR) method as a reference in determining methicillin resistance in Staphylococcus species, we aimed at comparing the reliability levels of disk diffusion, latex agglutination test and chromogenic agar use methods.

Methods: This prospective study was conducted on 228 Staphylococcus strains isolated between January 2020 and December 2020 in Samsun Training and Research Hospital. Disk diffusion, latex agglutination and chromogen agar medium methods were applied along with the polymerized chain reaction (PCR) method. Results: The mecA gene was detected in 47 of the isolates $(20.6 \%)$ by the PCR method, and these isolates were accepted as methicillin-resistant. When the PCR result was taken as a reference, the sensitivity of the disk diffusion method became $100 \%$, and specificity became $45.9 \%$; sensitivity of latex agglutination was determined as $80.9 \%$, and specificity as $70.2 \%$; sensitivity of chromogenic agar as $85.1 \%$ and its specificity was found to be $95 \%$. Only in S. aureus isolates, the highest sensitivity and specificity rate $(100 \%$ and $88 \%$, respectively) belonged to chromogenic agar.

Conclusion: Chromogenic agar provides more reliable data for $S$. aureus isolates, and the combined use of all three methods does not significantly increase reliability.
\end{abstract}

KEYWORDS: Staphylococcus, mecA, Chromogenic agar, Methicillin Resistance.

How to cite this:

doi: https://doi.org/10.12669/pjms.37.5.4274

Gorgun S, Isler H, Turgut MC. Comparison of rapid and conventional methods for investigating of mecA presence in Staphylococcus Species. Pak J Med Sci. 2021;37(5):1467-1474. doi: https://doi.org/10.12669/pjms.37.5.4274

This is an Open Access article distributed under the terms of the Creative Commons Attribution License (http://creativecommons.org/licenses/by/3.0), which permits unrestricted use, distribution, and reproduction in any medium, provided the original work is properly cited.

1. Selim Gorgun,

2. Hacer Isler,

3. Mehmet Cenk Turgut,

Department of Orthopedics and Traumatology,

Health Sciences University,

Regional Training and Research Hospital, Erzurum, Turkey.

1,2: Department of Microbiology and Clinical Microbiology,

Health Sciences University,

Samsun Training and Research Hospital,

Samsun, Turkey.

Correspondence:

Selim Gorgun, MD

Microbiology and Clinical Microbiology Specialist

Health Sciences University,

Samsun Training and Research Hospital,

Postal Code: 55090,

Samsun, Turkey.

E-mail: selimgorgun55@gmail.com

* Received for Publication:

* Revision Received:

* Revision Accepted: *

February 12, 2021

May 17, 2021

May 25, 2021

\section{INTRODUCTION}

Although Staphylococcus species are a member of the normal skin flora, they can cause a variety of infections ranging from simple wound infections to bacteremia and sepsis. Staphylococci are among the most common isolated agents in wound infections. The type of these agents and their resistance to antibiotics are of great importance for determining the treatment of infections..$^{1-3}$ Despite the fact that coagulase-negative Staphylococcus species cause more frequent infections, Staphylococcus aureus is known to be more pathogenic due to its various enzymes and factors. ${ }^{4,5}$

Methicillin resistance is the most important factor in the management of infections caused by Staphylococcus and the selection of antimicrobials. Methicillin-resistant Staphylococci are also 
resistant to beta-lactam group antibiotics in vivo, which significantly limits antibiotic options. ${ }^{4-6}$ In such cases, it is necessary to use antibiotic groups such as carbapenems, which leads to the development of significant resistance in some bacteria such as Acinetobacter and Pseudomonas that cause hospital infection. In such cases, antibiotics with a high rate of undesirable effects such as vancomycin or teicoplanin can also be an option. However, the development of resistance to this group of antibiotics, which is the most effective against Staphylococcus, constitutes one of the most dreadful scenarios in the world. ${ }^{4-8}$

Correct determination of the antibiotic resistance profile of Staphylococcus isolates obtained from the infection is critical for the management of the infection. Different methods have been tested for a long time to accurately determine whether there is methicillin resistance or not. Although molecular methods used for the detection of mecA and mecC genes that cause methicillin resistance are accepted as reference methods, for now, these methods cannot be used in routine applications. For this reason, the reliable detection of methicillin resistance by conventional methods has become a preferred necessity in terms of being both costeffective and easy to apply. ${ }^{4,7,9,10}$

In this study, we aimed at comparing the reliability levels of disk diffusion, latex agglutination test, and chromogenic agar methods, by taking the mecA gene detection by polymerized chain reaction (PCR) as a reference in determining methicillin resistance in Staphylococcus species.

\section{METHODS}

This study has been approved by the local ethics committee on 2020/13/8 and planned prospectively.

Obtaining and Identifying Strains: A total of 228 staphylococcal isolates obtained from various clinical samples sent to Samsun Training and Research Hospital's microbiology laboratory between January 2020 and December 2020 were included in the study. In the laboratory colony morphology, pigment formation, Gram's staining, catalase, and coagulase assays were concluded on the clinical specimens. Bacteriel identifications were detected by Vitec 2 (bioMérieux, France).

In the study, S. aureus ATCC 4103, S. aureus ATCC 25923, S. saprophyticus ATCC 45678, S. xylosus ATCC 95055, S. hominis ATCC 51624, S. capitis ATCC 56789, S. epidermidis ATCC 10003 were used as control strains.
Considering that it may belong to the same origin, only one of the isolates obtained from the same patient was included in the study. Similarly, only one of the isolates obtained from patients hospitalized in the same service (e.g. intensive care unit) on the same day was included in the study. Isolates that were not considered growth was far below the amount of a causative pathogen were excluded from the study.

\section{Determining Methicillin Resistance:}

Disc Diffusion Method: Cefoxitin susceptibility of Staphylococcus strains was determined by disc diffusion method. Discs containing $30 \mu \mathrm{g}$ Cefoxitin (Oxoid, Ireland) were used. For each strain, the solutions prepared with 0.5 McFarland turbidity were seeded on Mueller Hinton agar and the methicillin sensitivity was evaluated after 24 hours of incubation. ${ }^{11}$

Latex Agglutination: The presence of penicillinbinding protein $2 \mathrm{a}$ (PBP2a) in the isolates was determined using the Slidex MRSA kit (bioMérieux, France) and the test was carried out according to the manufacturer's recommendations. Positive control was used for each test.

Chromogenic Agar: In the study, chromID ${ }^{\circ}$ MRSA SMART kit (bioMérieux, France) was used, as well as a chromogenic medium used for screening methicillin resistance. The microorganism was cultivated on chromogenic agar under aseptic conditions. It required 18-24 hours of incubation. Methicillin-sensitive $S$. aureus (MSSA) could not grow on chromogenic agar, while methicillin-resistant $S$. aureus (MRSA) grown in a pinkish color. Other bacteria either could not reproduce or grow blue or colorless. ${ }^{12,13}$

MecA PCR: The multiplex PCR protocol used in the study was as follows: 10x PCR Buffer $2.5 \mu \mathrm{L}$, $10 \mathrm{mM}$ dNTP $0.5 \mu \mathrm{L}$, MECA 1 (10 pmol) $1.25 \mu \mathrm{L}$, MECA 2 (10 pmol) $1.25 \mu \mathrm{L}, 25 \mathrm{mM} \mathrm{MgCl} 23 \mu \mathrm{L}$, DNA Polymerase $0.5 \mu \mathrm{L}$, distilled water $13.5 \mu \mathrm{L}$ and bacterial DNA $2.5 \mu \mathrm{L}$. Thermal Cycler phase: 1 cycle at $94^{\circ} \mathrm{C}$ is 2 minutes, at $94^{\circ} \mathrm{C} 35$ cycles 15 sec, at $55^{\circ} \mathrm{C} 35$ cycles 30 seconds, $72^{\circ} \mathrm{C} 35$ cycles 30 seconds and $72^{\circ} \mathrm{C} 1$ cycle 10 minutes.

Statistical analysis: All statistical analyzes in the study were performed using SPSS 25.0 software (IBM SPSS, Chicago, IL, USA). Descriptive data were given in numbers and percentages.

Sensitivity, specificity, positive predictive value, negative predictive value, and accuracy of the methods were calculated. For sensitivity, the ratio of the number of isolates found to be resistant to methicillin to the actual number of resistant isolates, and for specificity, the ratio of the number of isolates found 


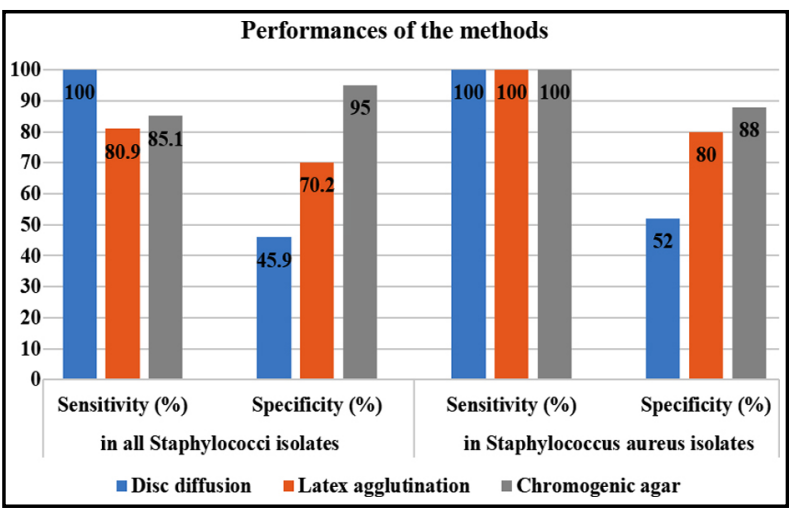

Fig.1: Sensitivity and specificity values of the methods.

to be susceptible to methicillin to the actual number of susceptible isolates was calculated. The positive predictive value was calculated from the ratio of the true resistant isolates among the isolates found to be resistant to methicillin, and the negative predictive value from the ratio of the actual susceptible isolates among the isolates found to be susceptible to methicillin. Finally, accuracy was calculated as the ratio of the total number of methicillin-resistant and susceptible isolates determined by the method to the total number of isolates.

\section{RESULTS}

In the study, $45.6 \%$ of the isolates were obtained from blood, $41.2 \%$ from wounded tissues, $11.4 \%$ from tracheal aspirate cultures, and $1.8 \%$ from cerebrospinal fluid. Of the isolates $34(14.9 \%)$ were S. aureus, 28 (12.3\%) were S. epidermidis, $23(10.1 \%)$ were S. hominis, 21 (9.2\%) were S. capitis, $20(8.8 \%)$

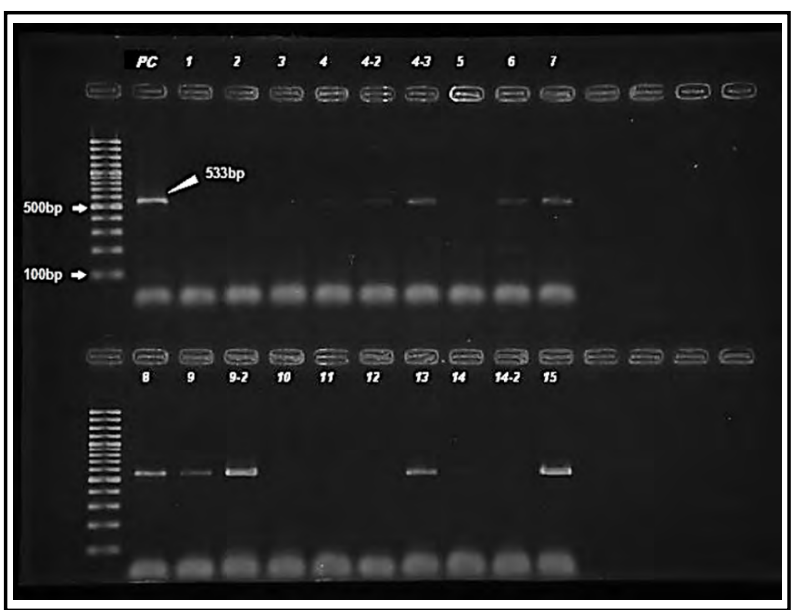

Fig.2: PCR amplification of MecA gene. Expected aplicon size is 533 bp. M 100 bp DNA ladder was used.

Lane 1 (PC) was the positive control.

were S. haemolyticus, 19 (8.3\%) were S. warneri and $83(36.4 \%)$ were other Staphylococcus species.

MecA gene was detected in 47 (20.6\%) of the isolates by using PCR method and these isolates were accepted as methicillin-resistant (Fig. 2). 145 (63.6\%) isolates were found to be methicillin-resistant by disk diffusion method, $92(40.4 \%)$ were found resistant by latex agglutination, and $49(21.5 \%)$ were found resistant by chromogenic agar (Table-I).

Only $S$. aureus isolates had the highest sensitivity and specificity rate for chromogenic agar $(100 \%$ and $88 \%$, respectively). The sensitivity of the chromogenic agar method in coagulase-negative Staphylococcus was $81.6 \%$ and its specificity was determined as $96.2 \%$ (Table-II) (Fig.1).

Table-I: Performance of three methods according to PCR result in all isolates.

\begin{tabular}{|c|c|c|c|c|c|c|c|c|}
\hline & \multicolumn{2}{|c|}{$P C R$} & \multirow{2}{*}{ Total } & \multirow{2}{*}{$\begin{array}{c}\text { Sensitivity } \\
(\%)\end{array}$} & \multirow{2}{*}{$\begin{array}{c}\text { Specificity } \\
(\%)\end{array}$} & \multirow{2}{*}{$P P V(\%)$} & \multirow{2}{*}{$N P V(\%)$} & \multirow{2}{*}{$\begin{array}{c}\text { Accuracy } \\
(\%)\end{array}$} \\
\hline & $R(n=47)$ & $S(n=18)$ & & & & & & \\
\hline Disc diffusion & & & & 100 & 45.9 & 32.4 & 100 & 57.0 \\
\hline $\mathrm{R}$ & 47 & 98 & 145 & & & & & \\
\hline S & 0 & 83 & 83 & & & & & \\
\hline Latex agglutination & & & & 80.9 & 70.2 & 41.3 & 93.4 & 72.4 \\
\hline $\mathrm{R}$ & 38 & 54 & 92 & & & & & \\
\hline S & 9 & 127 & 136 & & & & & \\
\hline Chromogenic agar & & & & 85.1 & 95.0 & 81.6 & 96.1 & 93.0 \\
\hline $\mathrm{R}$ & 40 & 9 & 49 & & & & & \\
\hline$S$ & 7 & 172 & 179 & & & & & \\
\hline
\end{tabular}

PCR: Polymerized chain reaction, PPV: Positive predictive value, NPV: Negative predictive value, S: Methicillin sensitive, R: Methicillin resistant. 
Selim Gorgun et al.

Table-II: Performance of three methods according to PCR result in Staphylococcus aureus isolates.

\begin{tabular}{|c|c|c|c|c|c|c|c|c|}
\hline & \multicolumn{2}{|c|}{ PCR } & \multirow{2}{*}{ Total } & \multirow{2}{*}{$\begin{array}{c}\text { Sensitivity } \\
(\%)\end{array}$} & \multirow{2}{*}{$\begin{array}{c}\text { Specificity } \\
(\%)\end{array}$} & \multirow{2}{*}{$\begin{array}{l}P P V \\
(\%)\end{array}$} & \multirow{2}{*}{$\begin{array}{c}N P V \\
(\%)\end{array}$} & \multirow{2}{*}{$\begin{array}{c}\text { Accuracy } \\
(\%)\end{array}$} \\
\hline & $R(n=9)$ & $S(n=25)$ & & & & & & \\
\hline Disc diffusion & & & & 100 & 52 & 42.9 & 100 & 64.7 \\
\hline $\mathrm{R}$ & 9 & 12 & 21 & & & & & \\
\hline S & 0 & 13 & 13 & & & & & \\
\hline Latex agglutination & & & & 100 & 80 & 64.3 & 100 & 85.3 \\
\hline $\mathrm{R}$ & 9 & 5 & 14 & & & & & \\
\hline$S$ & 0 & 20 & 20 & & & & & \\
\hline Chromogenic agar & & & & 100 & 88 & 75 & 100 & 91.2 \\
\hline $\mathrm{R}$ & 9 & 3 & 12 & & & & & \\
\hline$S$ & 0 & 22 & 22 & & & & & \\
\hline
\end{tabular}

PCR: Polymerized chain reaction, PPV: Positive predictive value, NPV: Negative predictive value, S: Methicillin sensitive, R: Methicillin-resistant.

The sensitivity and specificity of the chromogenic agar method were $100 \%$ and $88 \%$ for S. aureus, respectively; $100 \%$ and $100 \%$ for S. epidermidis and S. sciuri and $100 \%$ and $94.7 \%$ for S. hominis. The sensitivity and specificity of the chromogenic agar method were $100 \%$ and $88 \%$ for S. aureus, respectively; $100 \%$ and $100 \%$ for S. epidermidis and S. sciuri and $100 \%$ and $94.7 \%$ for S. hominis. The sensitivity and specificity of the latex agglutination method were determined as $100 \%$ and $94.4 \%$ for $S$. capitis, $100 \%$ and $89.5 \%$ for S. hominis. While most of the sensitivities of all three methods were found to be $100 \%$ for Staphylococcus species, specificity rates were observed to vary greatly. Among these common species, the median sensitivity of all three methods was $100 \%$. The specificity rates of disc diffusion were between $14.3 \%$ and $61.1 \%$ (median: $47.4 \%$ [interquartile range; IQR: $16.3 \%$ ]), and latex agglutination were between $50 \%$ and $94.4 \%$ (median: $76.9 \%$ [IQR: $28.0 \%$ ]), and chromogenic agar were between $75 \%$ and $100 \%$ (median: $100 \%$ [IQR: 12.0\%]). (Table-III).

Analysis was made considering the combination of methods, and the isolate detected by any method as susceptible to methicillin was considered susceptible. According to the analysis results, it was found that the combination of disk diffusion and chromogenic agar had the best performance with a sensitiv-

Table-III: Performance comparison of methods based on Staphylococcus species.

\begin{tabular}{lccccccc}
\hline & & \multicolumn{2}{c}{ Disc diffusion } & \multicolumn{2}{c}{ Latex agglutination } & \multicolumn{2}{c}{ Chromogenic agar } \\
\cline { 3 - 7 } & $n$ & $\begin{array}{c}\text { Sensitivity } \\
(\%)\end{array}$ & $\begin{array}{c}\text { Specificity } \\
(\%)\end{array}$ & $\begin{array}{c}\text { Sensitivity } \\
(\%)\end{array}$ & $\begin{array}{c}\text { Specificity } \\
(\%)\end{array}$ & $\begin{array}{c}\text { Sensitivity } \\
(\%)\end{array}$ & $\begin{array}{c}\text { Specificity } \\
(\%)\end{array}$ \\
\hline S.aureus & 34 & 100 & 52 & 100 & 80.0 & 100 & 88.0 \\
S.epidermidis & 28 & 100 & 53.8 & 100 & 76.9 & 100 & 100 \\
S.haemolyticus & 20 & 100 & 38.5 & 100 & 61.5 & 57.1 & 100 \\
S.capitis & 21 & 100 & 61.1 & 100 & 94.4 & 33.3 & 100 \\
S.sciuri & 19 & 100 & 14.3 & 100 & 50.0 & 100 & 100 \\
S.hominis & 23 & 100 & 47.4 & 100 & 89.5 & 100 & 94.7 \\
S.saprophyticus & 13 & 100 & 37.5 & 0,0 & 75.0 & 100 & 75.0 \\
Median & & 100 & 47.4 & 100 & 76.9 & 100 & 100 \\
Interquartile range & & 0 & 16.3 & 0 & 28.0 & 42.9 & 12.0 \\
\hline
\end{tabular}

Pak J Med Sci September - October 2021 Vol. $37 \quad$ No. $5 \quad$ www.pjms.org.pk 1470 
Table-IV: Isolates determined to be susceptible by any method are considered susceptible, performance of combinations of three methods according to PCR result in all isolates.

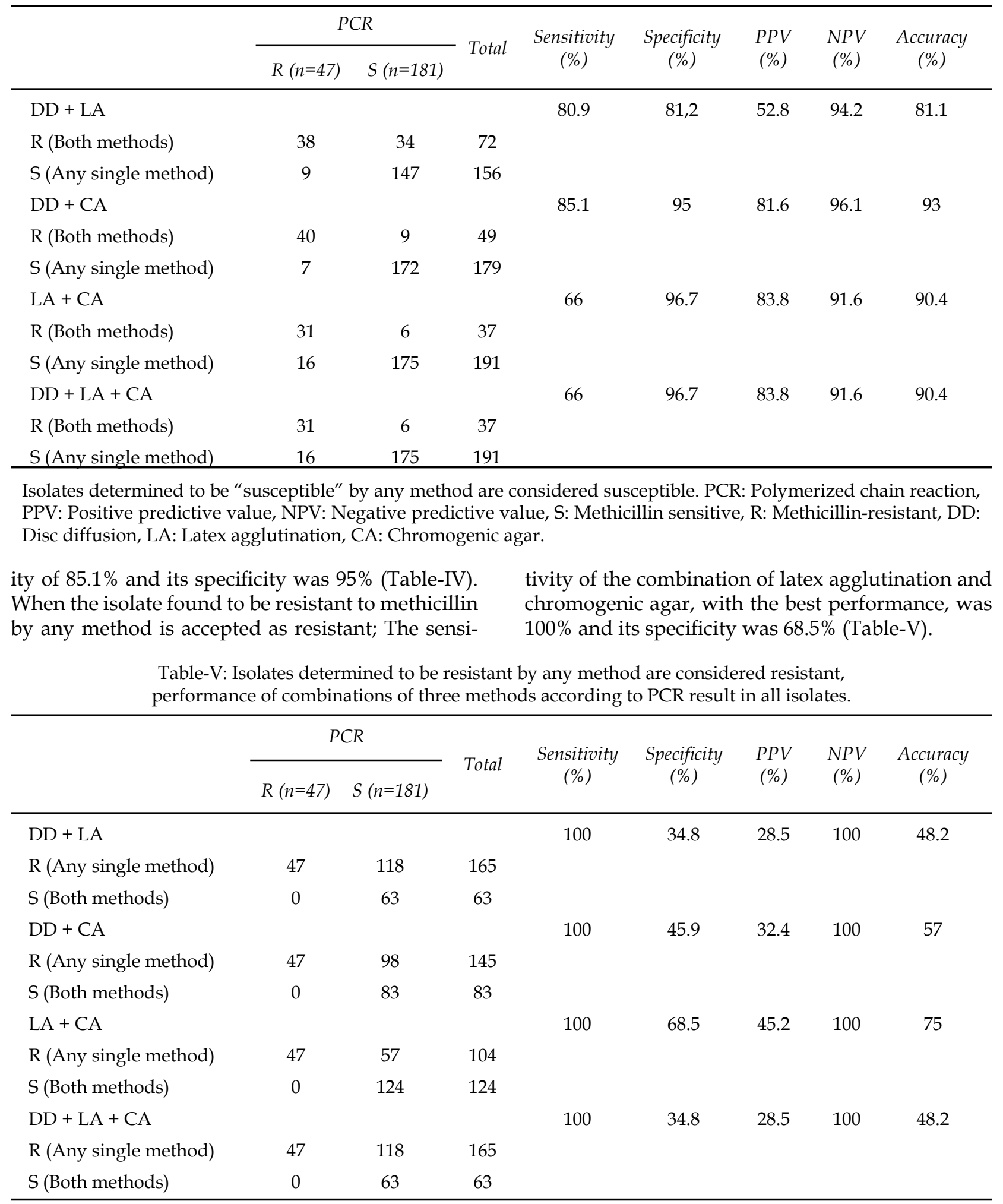

Isolates determined to be "resistant" by any method are considered resistant. PCR: Polymerized chain reaction, PPV: Positive predictive value, NPV: Negative predictive value, S: Methicillin sensitive, R: Methicillin-resistant, DD: Disc diffusion, LA: Latex agglutination, CA: Chromogenic agar. 


\section{DISCUSSION}

Staphylococcus is one of the bacteria isolated as the most common infectious agent and wound infection pathogen (between 19\% and 58.5\%). ${ }^{14-17}$ The presence of methicillin resistance is one of the most important guiding factors in the treatment of staphylococcal infections. Although some molecular methods and several dilution methods (for determining the minimum inhibitory concentration (MIC) value) have been developed to determine methicillin resistance in Staphylococcus species, they are not ideal for use in routine laboratory applications. ${ }^{4-7}$ Automated systems also determine methicillin resistance by determining the MIC value, but in many small-scale laboratories, these systems are not included for not being cost-effective. For these reasons, we focused on detecting methicillin resistance with easy and fast methods that can be applied in every laboratory. However, there remain uncertainties about whether these methods provide reliable data. ${ }^{4,7}$ The performance of three different methods was evaluated in our study.

One of the most classical methods for determining methicillin resistance in Staphylococcus is the disk diffusion method. Oxacillin has been used for a long time for this purpose. However, after it was determined that the reliability level was much lower than expected, oxacillin was replaced by cefoxitin, which gave better results. It has been reported that cefoxitin expresses the mecA better than other penicillins. ${ }^{18}$ However, the reliability of the disk diffusion method has been remaining under questioning so far. In some studies, it has been reported that the sensitivity of the disk diffusion test using cefoxitin disc is between $94 \%$ and $100 \%$ and the specificity is between $96 \%$ and $100 \%$ for determining methicillin resistance in Staphylococcus. ${ }^{19-24}$ When the PCR result is taken as a reference in this study, the sensitivity of the disk diffusion method in all Staphylococcus isolates was $100 \%$ and specificity was determined to be $45.9 \%$. Considering the highest number of isolated Staphylococcus in the study, the sensitivity of the disk diffusion method was $100 \%$ for all the isolates; also it was determined that the specificity rates varied between $14.3 \%$ and $61.1 \%$ (median: $47.4 \%$ [IQR: $16.3 \%]$ ). Accordingly, it was observed that the negative predictive value of the disk diffusion method was $100 \%$, but the positive predictive values were below $50 \%$. All these findings show that in Staphylococcus, the disk diffusion method, in general, captures methicillin resistance in almost all isolates. However, it is seen that the specificity rates for methicillin resistance are far below the acceptable level; that is, false-positive results are very high for methicillin resistance. This may cause the patient to use strong antibiotics unnecessarily when a methicillin-sensitive Staphylococcus is isolated as the agent. In the light of this, the disk diffusion method seems to be far from providing reliable data in both S. aureus isolates and coagulasenegative Staphylococcus.

MRSA isolates produce PBP2a, which has a lower affinity for beta-lactam antibiotics than PBP2. The mecA encodes PBP2a, which is the target of methicillin. ${ }^{12,20}$ The latex agglutination test used in our study is a serological method used for $S$. aureus isolates, determining the presence of $\mathrm{PBP} 2 \mathrm{a}$, and can be applied easily and quickly. In some studies, it has been reported that the sensitivity of the latex agglutination method using cefoxitin disc is between $88 \%$ and $100 \%$, and specificity is between $97 \%$ and $100 \%$ in determining methicillin resistance in Staphylococcus. ${ }^{12,20-22,25}$ In our study, the sensitivity of the latex agglutination method was found to be $80.9 \%$ and specificity was found to be $70.2 \%$ in all Staphylococcus isolates. Only in S. aureus isolates, the sensitivity and specificity rates were $100 \%$ and $80 \%$, respectively, and $76.3 \%$ and $68.6 \%$ for general coagulase-negative Staphylococcus, respectively. In most of the Staphylococcus species detected in high numbers in our study, the sensitivity of this method was determined as $100 \%$, whereas the specificity rates dispersed between $50 \%$ and $95 \%$. Specificity rates were found to be high in S. sciuri and S. hominis isolates, but methicillin resistance could not be determined in any of the S. saprophyticus isolates. These findings show that the latex agglutination method has a high sensitivity rate in determining methicillin resistance in general, its specificity for S. aureus is below the expected, and it is not usable in coagulase-negative Staphylococcus due to its highly unsteady performance.

The chromogenic agar used in our study is a medium that prevents the growth of methicillin-resistant Staphylococcus, because of the cefoxitin it contains. In addition, it indicates whether the growing colony is $S$. aureus or not based on the enzymes it contains. In this medium, the main purpose is to detect MRSA. ${ }^{12,13,26}$ In some studies, it has been reported that the sensitivity of the disk diffusion test using cefoxitin disc in determining methicillin resistance in Staphylococcus is between $75 \%$ and $100 \%$ and the specificity is between $89 \%$ and $100 \% .^{23,27-31}$ The sensitivity of chromogenic agar in our study 
was $85.1 \%$ and its specificity was determined as $95 \%$. The sensitivity and specificity rates were $100 \%$ and $88 \%$, respectively, in S. aureus isolates only; $81.6 \%$ and $96.2 \%$ in coagulase-negative Staphylococcus. Sensitivity and specificity rates were found to be $100 \%$ in some Staphylococcus species. Among these common species, the sensitivity [IQR: $42.9 \%$ ] and specificity [IQR: $12.0 \%$ ] median values of chromogenic agar were found to be $100 \%$. It was observed that the highest sensitivity and specificity rate for S. aureus isolates belonged to chromogenic agar among three methods. All these findings show that the use of chromogenic agar for the determination of methicillin in S. aureus isolates has yielded significantly reliable results, but its specificity is still somewhat low. According to these data, the reliability level of chromogenic agar generally gives relatively high results except for $S$. aureus.

In our study, in case the methods are used in combination, two separate analyzes were carried out. When the isolate determined by any method as susceptible to methicillin was considered sensitive, it was observed that the combination of disk diffusion and chromogenic agar showed the best performance with a sensitivity rate of $85.1 \%$ and the specificity rate of $95 \%$.

In cases where the methods were performed in combination, two analyses were carried out. When the isolate determined as resistant to methicillin by any method was considered as resistant, it was determined that the combination of latex agglutination and chromogenic agar showed the best performance, and the sensitivity was $100 \%$ and the specificity was $68.5 \%$. These findings show that the combined use of these methods either increases sensitivity or specificity but cannot increase both sensitivity and specificity. Accordingly, besides not providing high-reliability data, it is seen that their combined use does not significantly increase the level of reliability.

Limitations of the study: Since the study aimed at comparing routinely-used methicillin resistance detection methods, determination of minimum inhibitory concentration (MIC) values with seldomly used micro and macro dilution methods were not included. In addition, the presence of mecC genes was not investigated in the study. Methicillin resistance depends on the presence of the mecA or mecC genes, but the prevalence of mecC gene presence has been reported to be $0.009 \% 32$, and it was deemed that our study would not significantly affect the analysis results.

\section{CONCLUSION}

The data of our study show that disk diffusion, latex agglutination, and chromogenic agar methods do not yield high-reliability results in determining methicillin resistance in Staphylococcus species, but chromogenic agar provides more reliable data in $S$. aureus isolates, and the combined use of all three methods does not significantly increase the reliability level.

Acknowledgements: The authors are grateful to the editors and reviewers for their contributions to the study.

\section{Source of Funding: None.}

Conflicts of Interest: None.

\section{REFERENCES}

1. Wongkietkachorn A, Surakunprapha P, Titapun A, Wongkietkachorn N, Wongkietkachorn S. Periwound. Challenges Improve Patient Satisfaction in Wound Care. Plast Reconstr Surg Glob Open. 2019;7(3):e2134. doi: 10.1097/GOX.0000000000002134

2. Haalboom M, Blokhuis-Arkes MHE, Beuk RJ, Meerwaldt R, Klont R, Schijffelen MJ, et al. Culture results from wound biopsy versus wound swab: does it matter for the assessment of wound infection? Clin Microbiol Infect. 2019;25(5):629.e7-629.e12. doi: 10.1016/j.cmi.2018.08.012

3. Turhanoglu NM, Koyuncu E, Bayindir-Bilman F. Yara kulturlerinden izole edilen mikroorganizmalar ve antibiyotik dirençleri 2010-2015. Turk Hij Den Biyol Derg. 2018;75(2):183-194. doi: 10.5505/ TurkHijyen.2018.56338

4. Lee AS, de Lencastre H, Garau J, Kluytmans J, MalhotraKumar S, Peschel A, et al. Methicillin-resistant Staphylococcus aureus. Nat Rev Dis Primers. 2018;4:18033. doi: 10.1038/nrdp.2018.33

5. Monaco M, Pimentel de Araujo F, Cruciani M, Coccia EM, Pantosti A. Worldwide Epidemiology and Antibiotic Resistance of Staphylococcus aureus. Curr Top Microbiol Immunol. 2017;409:21-56. doi: 10.1007/82_2016_3

6. McCarthy H, Rudkin JK, Black NS, Gallagher L, $\mathrm{O}^{\prime}$ Neill E, O'Gara JP. Methicillin resistance and the biofilm phenotype in Staphylococcus aureus. Front Cell Infect Microbiol. 201528;5:1. doi: 10.3389/ fcimb.2015.00001

7. Ehlert K. Methicillin-resistance in Staphylococcus aureus -molecular basis, novel targets and antibiotic therapy. Curr Pharm Des. 1999;5(2):45-55.

8. Martins A, Cunha Mde L. Methicillin resistance in Staphylococcus aureus and coagulase-negative staphylococci: epidemiological and molecular aspects. Microbiol Immunol. 2007;51(9):787-795. doi: 10.1111/ j.1348-0421.2007.tb03968.x

9. Liu J, Chen D, Peters BM, Li L, Li B, Xu Z, et al. Staphylococcal chromosomal cassettes mec (SCCmec): A mobile genetic element in methicillin-resistant Staphylococcus aureus. Microb Pathog. 2016;101:56-67. doi: 10.1016/j.micpath.2016.10.028 
10. Lakhundi S, Zhang K. Methicillin-Resistant Staphylococcus aureus: Molecular Characterization, Evolution, and Epidemiology. Clin Microbiol Rev. 2018;31(4):e00020-18. doi: 10.1128/CMR.00020-18

11. The European Committee on Antimicrobial Susceptibility Testing EUCAST disc diffusion test manual. Version 6.0.2017 http://wwweucastorg/. Cited date January 2, 2021.

12. Tasse J, Dupieux C, Caillon J, Lanotte P, Lamy B, Aissa N, et al. Rapid bench identification of methicillin-sensitive and methicillin-resistant Staphylococcus aureus: A multicenter comparative evaluation of Alere PBP2a Culture Colony Test (Alere) Versus Slidex MRSA detection (bioMérieux). Diagn Microbiol Infect Dis. 2016;85(4):419421. doi: 10.1016/j.diagmicrobio.2016.04.008

13. Xu Z, Hou Y, Peters BM, Chen D, Li B, Li L, et al. Chromogenic media for MRSA diagnostics. Mol Biol Rep. 2016;43(11):1205-1212. doi: 10.1007/s11033-016-4062-3

14. Matkoski C, Sharp SE, Kiska DL. Evaluation of the Q score and Q234 systems for cost-effective and clinically relevant interpretation of wound cultures. J Clin Microbiol. 2006;44(5):1869-1872.

15. Sesli-Cetin E, Kaya S, Tas T, Cicioglu-Arıdogan B, Demirci M. Microorganisms Isolated from Surgical Site Infections and their Antibiotic Susceptibility Patterns. ANKEM Derg. 2006;20:89-93.

16. Demir H, Erandac M. Cerrahi alan infeksiyonlarından izole edilen mikroorganizmalar, Cumhuriyet Univ Tip Fak Derg. 2001;23(2):89-91.

17. Zafar A, Anwar N, Ejaz H. Bacteriology of infected wounds - A study conducted at Children Hospital Lahore. Biomedica. 2007;23:8(A):1-4.

18. Swenson JM, Fred CT, and the Cefoxitin Disk Study Group. Results of disk diffusion testing with cefoxitin correlate with presence of mecA in Staphylococcus spp. J Clin Microbiol 2005; 43: 3818-23.

19. Ahmad S. Detecting methicillin resistance in Staphylococcus aureus: comparison of different phenotypic methods and the polymerase chain reaction. Br J Biomed Sci. 2013;70(3):93-96. doi: 10.1080/09674845.2013.11669941

20. Akcam FZ, Tinaz GB, Kaya O, Tigli A, Ture E, Hosoglu $\mathrm{S}$. Evaluation of methicillin resistance by cefoxitin disk diffusion and PBP2a latex agglutination test in mecA -positive Staphylococcus aureus, and comparison of mecA with femA, femB, femX positivities. Microbiol Res. 2009;164(4):400-403. doi: 10.1016/j.micres.2007.02.012

21. Alipour F, Ahmadi M, Javadi S. Evaluation of different methods to detect methicillin resistance in Staphylococcus aureus (MRSA). J Infect Public Health. 2014;7(3):186-191. doi: 10.1016/j.jiph.2014.01.007

22. Naccache SN, Callan K, Burnham CA, Wallace MA, Westblade LF, Dien Bard J. Staphylococcus Ad Hoc Working Group of the CLSI Antimicrobial Susceptibility Testing Subcommittee. Evaluation of Oxacillin and Cefoxitin Disk Diffusion and Microbroth Dilution Methods for Detecting mecA -Mediated $\beta$-Lactam Resistance in Contemporary Staphylococcus epidermidis Isolates. J Clin Microbiol. 201922;57(12):e00961-19. doi: 10.1128/ JCM.00961-19

23. Uzun B, Karatas Sener AG, Gingor S, Afsar I, Yuksel Ergin O, Demirci M. Comparison of Cefoxitin Disk Diffusion Test, Automated System and Chromogenic Medium for Detection of Methicillin Resistance in Staphylococcus aureus Isolates. Mikrobiyol Bul. 2013;47(1):11-18. Turkish. doi: $10.5578 / \mathrm{mb} .4304$
24. Demir T, Coplu N, Esen B. Comparative analysis of phenotypic and genotypic detection of methicillin resistance among Staphylococcus aureus. Indian J Pathol Microbiol. 2016;59(3):314-317. doi: 10.4103/03774929.188103

25. Andriesse GI, Elberts S, Vrolijk A, Verhulst C, Kluytmans JA. Evaluation of a fourth-generation latex agglutination test for the identification of Staphylococcus aureus. Eur J Clin Microbiol Infect Dis. 2011;30(2):259-264. doi: 10.1007/ s10096-010-1080-2

26. Yagmur G, Ercal BD, Mengeloglu Z, Sariguzel FM, Berk E, Saglam D. Is rapid antibacterial susceptibility testing medium reliable for routine laboratory practices? Pak J Med Sci. 2015;31(2):351-354. doi: 10.12669/pjms.312.6683

27. Kluytmans J, Van Griethuysen A, Willemse P, Van Keulen P. Performance of CHROMagar selective medium and oxacillin resistance screening agar base for identifying Staphylococcus aureus and detecting methicillin resistance. J Clin Microbiol. 2002 Jul;40(7):2480-2. doi: 10.1128/jcm.40.7.2480-2482.2002.

28. Diederen B, van Duijn I, van Belkum A, Willemse P, van Keulen P, Kluytmans J. Performance of CHROMagar MRSA medium for detection of methicillin-resistant Staphylococcus aureus. J Clin Microbiol. 2005;43(4):19251927. doi: 10.1128/JCM.43.4.1925-1927.2005

29. Han Z, Lautenbach E, Fishman N, Nachamkin I. Evaluation of mannitol salt agar, CHROMagar Staph aureus and CHROMagar MRSA for detection of methicillin-resistant Staphylococcus aureus from nasal swab specimens. J Med Microbiol. 2007;56(Pt-1):43-46. doi: 10.1099/jmm.0.46777-0

30. van Hal SJ, Stark D, Lockwood B, Marriott D, Harkness J. Methicillin-resistant Staphylococcus aureus (MRSA) detection: comparison of two molecular methods (IDIMRSA PCR assay and GenoType MRSA Direct PCR assay) with three selective MRSA agars (MRSA ID, MRSASelect, and CHROMagar MRSA) for use with infection-control swabs. J Clin Microbiol. 2007;45(8):2486-2490. doi: 10.1128/ JCM.00139-07

31. Nahimana I, Francioli P, Blanc DS. Evaluation of three chromogenic media (MRSA-ID, MRSA-Select and CHROMagar MRSA) and ORSAB for surveillance cultures of methicillin-resistant Staphylococcus aureus. Clin Microbiol Infect. 2006;12(12):1168-1174. doi: 10.1111/j.1469-0691.2006.01534.x

32. Diaz R, Ramalheira E, Afreixo V, Gago B. Methicillinresistant Staphylococcus aureus carrying the new mecC gene--a meta-analysis. Diagn Microbiol Infect Dis. 2016;84(2):135-140. doi: 10.1016/j.diagmicrobio.2015.10.014

\section{Author Contribution:}

SG, HI, MCT: Conceptualization and design of study.

SG, HI: Methodology, takes the responsibility for integrity of the study.

SG, HI, MCT: Data processing. SS, MCT: Statistical Analyse.

SG, HI, MCT: Writing-editing. 